\title{
Special issue on 10 years of cognitive radio: state-of-the-art and perspectives
}

\author{
Jacques Palicot ${ }^{1 *}$, Joseph Mitola III' , Zander (Zhongding) Lei $^{3}$ and Friedrich K Jondral ${ }^{4}$
}

Cognitive radio (CR) is a technology that is of growing interest internationally. Currently, there is a highly active worldwide community of academic and industrial researchers working in this field. Significant efforts have been made and focused on the many technical questions as well as on the economic and political challenges in order to move this new technology from research concept to reality.

A CR is a radio that is able to behave somehow as a cognitive system, having at least the capabilities of observing, making decisions and adapting, i.e., the three main functions of the simplified cognitive cycle.

CR technologies, such as Dynamic Spectrum Access (DSA), create huge opportunities for interesting research and development in a wide range of applications. Since 1999, CR received a lot of attention especially in spectrum optimization. Currently, technical efforts and standardization are focused on UHF spectrum and TV bands. TV signals are easy to detect and are quasi-static in the sense that transmitters' locations and frequencies are fixed. Furthermore, due to the allocation and frequency reuse in order to protect against interferences, typically there is a lot of unused spectrum at a specific location. This free spectrum is known as "White Spectrum" or "White Space". CR has been often reduced to the use of white space by DSA, but CR could be envisaged in a much wider sense, depending on which types of sensors are considered. At a larger scale, CR gives equipments smartness that can be exploited at every level of the equipment, in order to make it more efficient for the network and more helpful to the user. A CR system may manage its power resources, anticipate its environment changes, make networks vertical handover, etc. Cognitive networks aim at improving system capacity, thanks to $\mathrm{CR}$ devices providing enhanced spectrum sharing capabilities, a better quality of service and performance.

\footnotetext{
* Correspondence: jacques.palicot@supelec.fr

${ }^{1}$ IETR/Supelec, Campus de Rennes Avenue de la Boulais, BP 81127,

Cesson-Sévigné Cedex 35511, France

Full list of author information is available at the end of the article
}

After 10 years of $\mathrm{CR}$, this special issue aims at putting together an overview of the major achievements and developments in this field, and showing the most important challenges that remain in CR. There are 19 papers in this Special Issue, which has assembled papers dealing with the three main functions of the simplified cognitive cycle. As it can be seen in the following figure, eight papers are dealing with sensing at large; four with decision making and learning; three papers with adaptation in the sense of reconfigurable hardware for $C R$ receivers or insertion in the spectrum; three papers address application of CR; and finally, one other is dealing with another very important aspect of CR: security (See Figure 1).

Among the eight papers dealing with sensing, two papers are providing a review of 10 years of spectrum sensing studies. The paper "Ten years of research in spectrum sensing and sharing in cognitive radio" by $\mathrm{L} \mathrm{Lu}$, $\mathrm{X}$ Zhou, U Onunkwo, G Li provides an overview of recent research achievements, including spectrum sensing, sharing techniques, and the applications of $\mathrm{CR}$ systems. It gives a very broad and complete overview of sensing techniques (both classical and advanced) in many contexts (local, distributed, cooperative, etc.). This overview incorporates 140 references which cover the entire topic. The paper "A survey on dynamic spectrum access protocols for distributed cognitive wireless networks", by P Ren, Y Wang, Q Du, J Xu, proposes a classification for DSA protocols based on different criteria, such as spectrum sharing modes, spectrum allocation behaviors, spectrum access modes, the usage of common control channels, spectrum usage strategies, the number of radios, and spectrum sensing techniques. Following this classification, they propose three categories of protocols: contentionbased, time-slotted, and hybrid protocols. They conclude their article with a description of open issues.

The six remaining papers in this sensing category deal with several very different aspects of sensing from very theoretical to more applicative aspects. The paper "Noncommutative large entries for cognitive radio applications" 


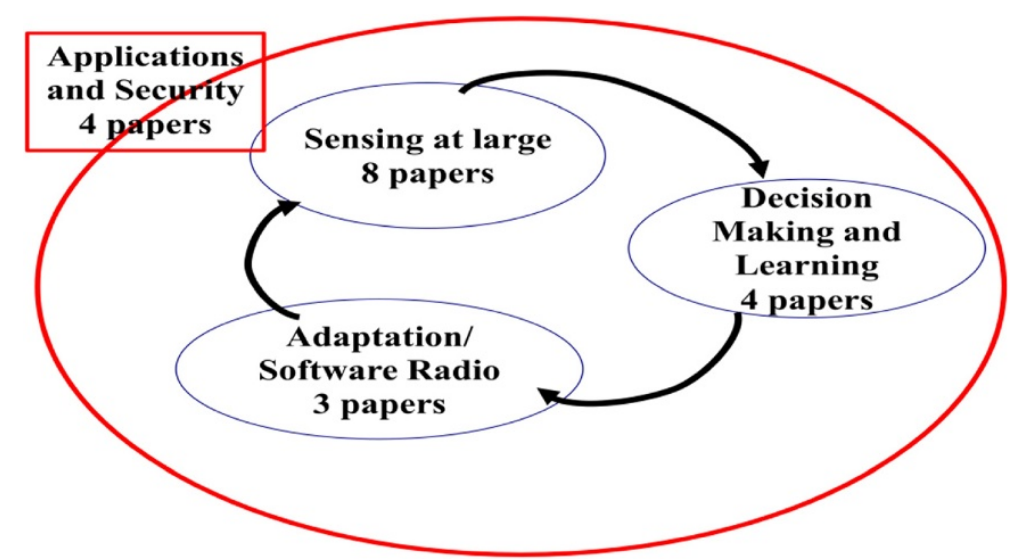

Figure 110 year of Cognitive Radio: State of the Art and Perspective 19 papers.

by AM Masucci and M Debbah addresses the problem of eigen-inference using advanced tools of free probability theory, an extension of classical probability theory to noncommutative algebras. This framework has many applications in signal detection and sensing when the number of received samples is of the same order as the dimension (time, frequency, or space) of the observed vector. The paper "Dimension estimation-based spectrum sensing for cognitive radio" by B Zayen and A Hayar exploits signal space analysis and presents a dimension estimation-based detector for sensing in CR context. The proposed algorithm is formulated as a binary hypothesis test using Akaike information criterion and the minimum description length criterion. Closed-form expressions of false alarm probabilities are derived for a given threshold. The paper "Spectrum sensing for cognitive radio exploiting spectrum discontinuities detection" by W Guibene, M Turki, B Zayen and A Hayar presents an algebraic framework to model spectrum discontinuities for sensing in wideband CR systems. The information derived at the level of these irregularities is exploited in order to derive a new spectrum sensing algorithm that is able to locate the borders of occupied bands. In the paper "Statistical analysis of linear spatial holes estimators in cognitive radio" by M Kazemi, M Ardebilipour and B Mahboobi, an important linear spatial hole estimation algorithm is studied in CR systems: the received signal strength algorithm based on weighted least square (RSS-WLS). In particular, a closed form expression for mean and covariance of estimation of the RSS-WLS algorithm is derived. Performance is compared with the Cramer Rao lower bound where it is shown that cognitive RSS-WLS estimator is asymptotically efficient. In the paper "Optimization of cooperative spectrum sensing with sensing user selection in cognitive radio networks" by $\mathrm{H} \mathrm{Yu}, \mathrm{W}$ Tang and $\mathrm{S} \mathrm{Li}$, the authors are interested in cooperative spectrum sensing. They particularly focus on the delay of the secondary user
(SU) for reporting their local sensing data to the fusion center. They formulate the optimization problem considering the delays in both Gaussian and Rayleigh fading conditions. The last paper in the sensing category is the paper "Blind recovery of $k / n$ rate convolutional encoders in a noisy environment" by M Marazin, R Gautier and G Burel. This paper deals with signal feature detection. The feature considered is the type of channel code, or more precisely the parameters and generator matrices of convolutional encoders. The authors claim that an intelligent receiver should be able to adapt itself to a specific transmission context. A new iterative method is developed dedicated to the blind estimation of convolutional encoders in a noisy context and its performance is studied.

Among the four papers dealing with Decision Making and Learning, two papers are addressing the state-of-the-art of CR. As a matter of fact, both papers provide a review of the first 10 years of decision making and learning tools applied to CR. On the one hand, the paper "Decision making for cognitive radio equipment: analysis of the first 10 years of exploration" by W Jouini, C Moy and $\mathrm{J}$ Palicot draws a general retrospective view on the first 10 years of $\mathrm{CR}$ from the decision making and learning point of view from an equipment perspective. A design space and a classification based on the " $a$ priori knowledge" notion have been defined in order to discriminate existing methods in the literature. The impact of sensing errors on the decision-making process is also discussed in this paper. On the other hand, the paper "An overview of learning mechanisms for cognitive systems" by A Bantouna, V Stavroulaki, Y Kritikou, K Tsagkaris, P Demestichas and $\mathrm{K}$ Moessner presents in brief the state-of-the-art of machine learning in cognitive systems as coming from the recent research with an overview of three different learning capabilities suggested for both the network and the user device. The paper "CR engine parametric optimization utilizing Taguchi analysis" by AE Amanna, 
D Ali, M Gadhiok, M Price and JH Reed deals with a formalized process using Tagachi method and orthogonal testing arrays in order to identify parameters settings for genetic algorithms for the Cognitive Engine. $\mathrm{O}$ van den Biggelaar, JM Dricot, P De Doncker and F Horlin in their paper "Sensing time and power allocation for cognitive radios using distributed Q-learning" aim to illustrate the potential of Q-learning for CR systems. They propose two decentralized resource allocation Q-learning algorithms: the first is used to share the sensing time among the CRs in a way that maximizes the throughput of the radios. The second is used to allocate the CR power in a way that maximizes the signal to interference-plus-noise ratio at the secondary receivers while meeting the primary protection constraint.

Concerning papers related to the adaptation function of the cognitive cycle, the first paper "Multistage quadrature sigma-delta modulators for reconfigurable multiband analog-digital interface in cognitive radio devices" by J Marttila, M Allén and M Valkama deals with the enabling technology which will offer $\mathrm{CR}$ reconfigurable hardware via Software Radio technology. The authors propose a flexible and reconfigurable A/D interface for $\mathrm{CR}$ receivers enabling effcient parallel reception of multiple noncontiguous frequency slices. This is based on multistage quadrature $\Sigma \Delta$ modulator $(\mathrm{Q} \Sigma \Delta \mathrm{M})$ concept; its performance is analyzed. The second paper, "Capacity bounds for multiple access-cognitive interference channel" by M Mirmohseni, B Akhbari and MR Aref, studies the capacity regions at the Media Access Control level, by proposing the Multiple Access-Cognitive Interference Channel in a scenario with cognitive transmitters and a Multiple Access Channel with common information that enables cooperation among them. The third paper, "Protection of primary users in dynamically varying radio environment: practical solutions and challenges" by P Kryszkiewicz, H Bogucka and AM Wyglinski, presents non-contiguous orthogonal frequency division multiplexing as a promising and practical approach for achieving spectrally agile wireless data transmission that is suitable for SUs to access fragmented spectral opportunities more efficiently. The insertion of cancellation carriers and windowing permits to insert the spectrum with very low out-of-band radiation in order to protect signals of the primary users (PUs) after insertion.

Among the papers which are dealing with applications of CR and with others aspects of CR, paper "Security in cognitive wireless sensor networks. Challenges and open problems" by A Araujo, J Blesa, E Romero and D Villanueva deals with the security aspect, which is a major challenge in cognitive wireless sensor networks, and more generally for $\mathrm{CR}$. This article discusses a wide variety of attacks on cognitive wireless sensor networks, their taxonomy and the different security measures available to handle the attacks.
The paper "Adaptive cognitive media delivery over composite wireless networks" by $\mathrm{T}$ Farnham proposes to deliver multimedia content by adapting to the available resources in an opportunistic manner. This is obtained by applying cognitive resource management using distributed decision making between devices. $\mathrm{H}$ Peng and T Fujii in their paper "Joint cross-layer resource allocation and interference avoidance with Quality of Service (QoS) support for cognitive radio systems" study the coexistence and optimization of a centralized orthogonal frequency-division multiple access-based multiuser $C R$ system which coexists with a cellular primary system. They define the relationship between power limits at SUs and interference margin at PUs. To provide the SUs with satisfactory QoS and the sum rate of the multiuser CR system, they propose a constrained two-variable nonlinear optimization problem, which is solved using the Lagrangian duality based technique with iterative water-filling to find the optimal power and subcarrier allocation. The last paper in this application category, "Advances in opportunistic radio technologies for TVWS" by D Noguet, M Gautier and V Berg, considers the most recent regulatory rules for TV White Space opportunistic usage, and proposes technologies to operate in these bands. Spectrum-sensing performance is discussed with respect to TV White Space regulation parameters. Modulation schemes for the opportunistic radio are discussed, showing the limitations of classical multi-carrier techniques and the advantages of filter bank modulations; in particular, the low adjacent band leakage of filter bank is addressed, and its benefit for spectrum pooling is stressed as a means to offer broadband access through channel aggregation.

\section{Competing interests}

The authors declare that they have no competing interests.

\section{Authors' information}

Guest Editors: Dr. Joseph Mitola III, Dr. Zander (Zhongding) Lei, Prof. Dr.rer. nat. Friedrich K. Jondral.

Prof. Jacques PALICOT: SUPELEC/Institut d' Electronique et de

Télécommunications de Rennes France. Prof. Jacques Palicot received, in 1983, his PhD degree in Signal Processing from the University of Rennes. Since 1988, he has been involved in studies about equalization techniques applied to digital transmissions and new analog TV systems. Since 1991 he has been involved mainly in studies concerning the digital communications area and automatic measurements techniques. He has taken an active part in various international bodies EBU, CCIR, URSI, and within RACE, ACTS and IST European projects. He has published various scientific articles notably on equalization techniques, echo cancellation, hierarchical modulations and Software Radio techniques. He is currently involved in adaptive Signal Processing and in new techniques as Software Radio and Cognitive radio. From November 2001 to September 2003 he had a temporary position with INRIA/IRISA in Rennes. He serves as Associate Editor for EURASIP JASP since 2008. He also served as lead guest editor several Special Issues on Software Radio, Cognitive Radio and Green Radio. He was Technical Program Chairman of CROWNCOM 2099 and Co General Chairman of ISCIT 2011. Since October 2003 he is with Supélec in Rennes where he leads the Signal Communications and Embedded Electronics (SCEE) research team.

Dr. Joseph Mitola III: Founder and CEO, Mitola's STATISfaction, Saint Augustine, Florida, USA. Dr. Mitola received his B.S. in Electrical Engineering 
(Highest Honors) from Northeastern University, Boston in 1971; M.S.E from The Johns Hopkins University, Baltimore in 1974; and the Licentiate and Doctorate in Teleinformatics from KTH, The Royal Institute of Technology, Stockholm in 1999 and 2000. Widely cited, Dr. Mitola is recognized globally for his seminal contributions to software radio and cognitive radio; Mitola's STATISfaction extends CR via statistics and other mathematical techniques to enhance user satisfaction with personal information systems. Previously, Dr. Mitola was Vice President for the Research Enterprise of Stevens Institute of Technology. During his 15 years at The MITRE Corporation (1993-2008), he served as Joint Special Assistant to the Director of the US Defense Advanced Research Projects Agency (DARPA) and to the Deputy Director of the US National Security Agency (NSA) for Trustable Cognitive Systems as well as Chief Scientist of the DoD Federally Funded Research and Development Center. He also has held positions of technical leadership with ITT Corporation, E-Systems, Advanced Decision Systems, and Harris Corporation. He was founding chair of the SDR Forum.

Dr. Zander (Zhongding) Lei: Institute for Infocomm Research, Singapore. Dr. Zander (Zhongding) Lei received his B.S. (Honor, 1st upper) and the Ph.D degrees from Beijing Jiaotong University, Beijing, China in 1991 and 1997, and the M.Eng degree from Tong Ji University, Shanghai, China in 1994. From 1997 to 1999, he was a Post-doctoral Fellow in the National University of Singapore. Since 1999, he has been with Institute for Infocomm Research Singapore, where he is a Research Scientist and Project Manager, leading teams working on next generation cellular and local area networks. He is a technical leader in IEEE 802.22 standard for wireless regional area network, published the first IEEE standard for CR systems. His research interests include next generation/emerging wireless communication systems/ technologies, cooperative communications, CR etc. He has 9 patents granted and has published over 90 papers in international refereed journals or conferences. Dr. Lei is a co-recipient of Institution Engineering of Singapore Prestigious Engineering Achievement Awards in both 2005 and 2007. He has been the PHY Technical Editor for IEEE 802.22 standard since 2006. He is an Editor for IEEE Communications Letters and IEEE Communications Magazine, a Guest Editor for IEEE Wireless Communication Magazine and a Lead Guest Editor for EURASIP WCN Special Issue on Multicell Cooperation.

Prof. Friedrich K. Jondral: Karlsruhe Institute of Technology (KIT), Karlsruhe, Germany. Prof. Friedrich K. Jondral received a Diploma in mathematics and a Doctoral degree in natural sciences from the Technische Universität Braunschweig, Germany, in 1975 and 1979, respectively. From 1979 to 1992 Dr. Jondral was an employee of AEG-Telefunken (now Cassidian Electronics), Ulm, Germany, where he held various research, development and management positions. During this period he also lectured on applied mathematics at the Universität UIm where he was appointed Adjunct Professor in 1991. Since 1993 Dr. Jondral has been Full Professor at the Karlsruhe Institute of Technology, Germany, and Director of the Communications Engineering Lab. Here, from 2000 to 2002, he served as the Dean of the Department of Electrical Engineering and Information Technology. His current research interests are in the fields of ultra wideband communications, software defined and cognitive radio, signal analysis, pattern recognition, network capacity optimization and dynamic channel allocation.

\section{Acknowledgments}

The four guest editors would like to thank the authors of all submitted papers (both those that were accepted and those, unfortunately, that could not) for considering our special issue to disseminate their work. We also would like to warmly thank all the reviewers for their difficult and conscientious work and for the time spent for their review. We extend our thanks to JWCN staff mainly Bernardino McCartney and to the JWCN editor in Chief Luc Vandendorpe to offer us the opportunity to propose this Special Issue. Finally, the guest editors hope that the readers can use efficiently the research work presented in these 19 papers to extend CR studies and applications for the next 10 years at least.

\section{Author details}

${ }^{1}$ IETR/Supelec, Campus de Rennes Avenue de la Boulais, BP 81127, Cesson-Sévigné Cedex 35511, France. ${ }^{2}$ Stevens Institute of Technology, Castle Point on Hudson, Hoboken, NJ, USA. ${ }^{3}$ Technical Editor for IEEE 802.22 WRAN Standard, Institute for Infocomm Research (I2R), Fusionopolis Way, \#21-01, Connexis, 138632, Singapore. ${ }^{4}$ Karlsruhe Institute of Technology (KIT), Communications Engineering Lab (CEL), Karlsruhe 76128, Germany.

Received: 29 May 2012 Accepted: 29 May 2012

Published: 12 July 2012

doi:10.1186/1687-1499-2012-214

Cite this article as: Palicot et al: Special issue on 10 years of cognitive radio: state-of-the-art and perspectives. EURASIP Journal on Wireless Communications and Networking 2012 2012:214

\section{Submit your manuscript to a SpringerOpen ${ }^{\odot}$ journal and benefit from:}

- Convenient online submission

- Rigorous peer review

- Immediate publication on acceptance

- Open access: articles freely available online

- High visibility within the field

- Retaining the copyright to your article

Submit your next manuscript at $>$ springeropen.com 